he needs capital for a bigger, more-fun computer, he must cite the research of his parasites. The true host is happy when his computer is overloaded because he wanted a bigger one anyway, and the overload gives him the excuse to buy the machine he really wants. The pathway to a new computer is the ultimate benefit a parasite can confer on a host.

A third advantage for the host is that the parasite may write programs that are available to the host. Actually, this is irrelevant. If he likes a parasite's program, a host will write his own equivalent version. Other people's programs are never adequate for a true host, and this dissatisfaction extends to system programs as well.

The final basic principle of parasitism is never to pain a host unnecessarily. When stealing time, you must do it painlessly. For example, at all costs try to minimize the frequency of your name on the schedule sheets; just appear when the computer is vacant. Of course, every species of parasite has developed its own devious ways of camouflaging its activity; adaptation to local conditions is the best advice.

In all fairness, I must say that at the Bell Telephone Laboratories I have been fortunate to find a most generous

\title{
The description and luminous calibration of cathode ray oscilloscope visual displays*
}

\author{
GEORGE SPERLING† \\ Bell Telephone Laboratories, Murray Hill, New Jersey 07974 \\ and \\ New York University, New York, New York 10003
}

A description of a CRO display should include descriptions of: (1) typical display contents (e.g., a photograph), (2) CRO output parameters (e.g., refresh rate), and (3) luminous measurements. Luminous calibrations are unorthodox because CRO displays are discontinuous in space and in time, and because they are sources, not reflectors, of light. The appropriate luminous quantities are luminous intensity and the integral of luminous intensity (luminous directional energy, $L D E$ ); the appropriate measurements are of $L D E$ per point and of $L D E$ per unit line length. A simple calibration procedure is described, and the formulas relating these quantities to luminances are given.

A description of a cathode ray oscilloscope (CRO) visual display should be sufficiently detailed to enable a reader to produce a visually

*Originally presented at the North Atlantic Treaty Organization, Advanced Studies Institute on: On-Line Computing for Behavioral Science, Department of Psychology, Sheffield, England, July 19, 1969. host, Peter Denes. He is difficult to get along with, but so am I, and the fact that we have had successful interaction for over 3 years points to the great stability of the parasite-host to be the greatest advantage of the parasite-host relation. The parasite thinks that when the host finally discards the computer, he, the parasite, can buy the depreciated computer at a bargain price. Of course, you immediately recognize the fallacy: when he does this, the parasite becomes the host-the ultimate catastrophe.

If ever you are presented with a computer, you must search for a host-a larval host who has not yet found his own computer. Like nature, the computer universe abounds with prospective hosts. Once you have discovered a larval host and brought him near the computer, he'll metamorphose and build his nest there. And you will continue to be able to enjoy the use of the computer for research without knowing anything about how it works inside. This is the ultimate application of behavioral science.

\section{NOTE}

1. The second alternative will be dismissed later. relationship. This stability may seem

described by a photograph of a typical frame with sufficient resolution to define the points, vectors, characters, or scan lines of which the frame is composed. The photograph should contain a size reference and an insert enlargement, if necessary, to show the point or line structure. The accompanying description should include a statement of the viewing distance. Black-on-white pictures reproduce well with ordinary printing methods, whereas white-on-black pictures generally require expensive photographic reproduction to produce acceptable copies. Therefore in preparing figures, it often is desirable to use a film that gives positive transparencies directly (e.g., Polaroid $146 \mathrm{~L}$ ) so that the resulting prints will be negatives of the display.

The description of the output parameters should include the refreshment rate, the rate of plotting points or characters, the total time taken to paint a frame, and the phosphor's time constant and chromaticity (e.g., color temperature, if known).

Luminous measurements should include the luminous directional energy per point of intensified points (explained below), the unintensified background screen luminance, and the approximate room illuminance.

It is in their treatment of luminous quantities that most CRO descriptions fail. Difficulties in luminous calibrations of CRO displays arise for two reasons: (1) whereas most natural objects are reflectors of light and best characterized by their reflectances or luminances, CRO displays are sources of light and usually are best characterized by their intensities or directional energies; and (2) whereas most naturally occurring objects are illuminated continuously in time and their luminance changes relatively continuously in space, CRO displays typically are pulsed at discrete instants in time and at discrete points in space. Calibration difficulties are overcome by measuring the appropriate luminous quantities of CRO displays, namely the luminous directional energy per point and the luminous directional energy per line length. The measurement techniques are described below.

equivalent display, either on a CRO or by nther means. Such a description need not be insufferably detailed; on the contrary, by judicious selection of the relevant aspects it can be both short and useful. The description should include three kinds of information: (1) a description of the display contents; (2) a description of the CRO output parameters (refresh rate, etc.); and (3) luminous measurements.

The display contents are best

\section{LUMINOUS DIRECTIONAL ENERGY PER POINT}

\section{Point Source}

A visual point source is defined as a luminous area whose maximum diameter is sufficiently small that the visual response does not depend on the actual distribution of light within the area, but only on the total flux. The largest allowable diameter for a point source varies with overall illumination and with the direction of view, being 


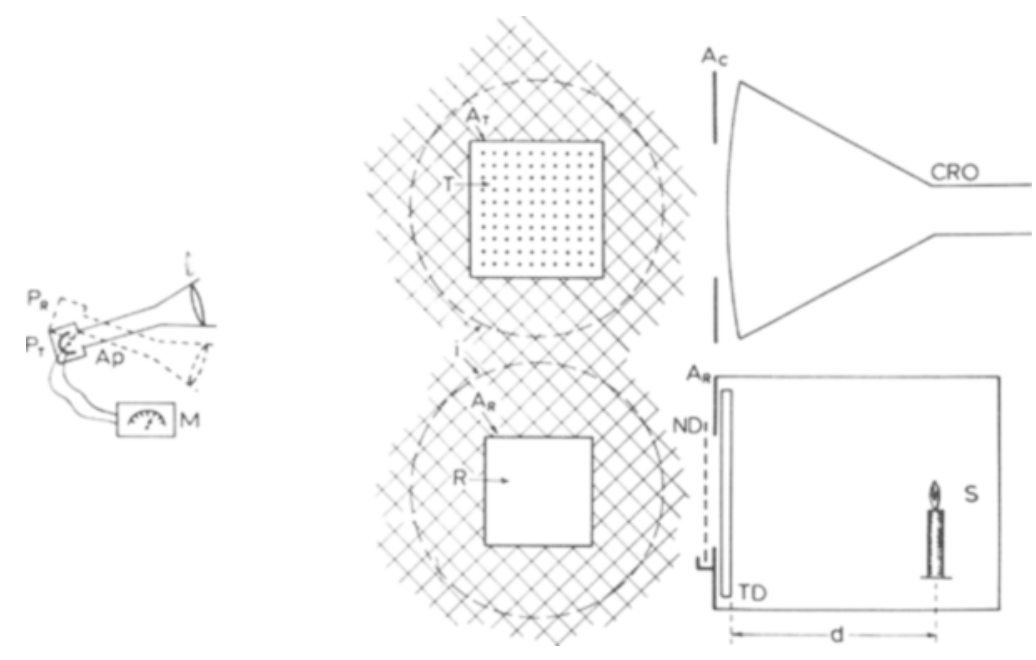

Fig. 1. Reference source (R) and CRO test pattern (T) for calibration of luminous energy per point. The reference surface of known luminance $(R)$ is seen through the aperture $A_{R}$; it is formed by a source of known candle power (S) illuminating a plate of uniformly transmitting-diffusing glass (TD). The reference luminance may be varied by altering the distance (d) or by interposing neutral density filters (ND). The test pattern is composed of a 10 by 10 point matrix, seen through an aperture $A_{T}$ that excludes all other points. The luminous intensity of $T$ relative to $R$ is determined by a photometer which may be in one of two positions: $P_{R}$ and $P_{T}$. The photometer consists of a lens $(\ell)$, an aperture (Ap), a photodetector $(\subset)$, and a meter $(M)$. The conjugate image of the smaller of the aperture, Ap, and the photosensitive surface, $\leftarrow$, is the acceptance region (i) of the photometer and is shown as a dotted circle against $A_{R}$ and $A_{T}$.

smallest in the 2 deg of central vision. As a rule of thumb, $4 \mathrm{~min}$ of arc (about $1 \mathrm{~mm}$ at $1 \mathrm{~m}$ viewing distance) may be taken as the limiting size of a visual point source. When a source is bigger than about $4 \mathrm{~min}$, the spatial distribution of light as well as the total intensity must be given. The spot diameter of a CRO beam (plus the viewing distance) usually is a sufficient spatial characterization; this is given implicitly in a photograph of the display.

For photometric purposes, "photometric point sources" can be much bigger; even $20 \mathrm{deg}$ is permissible when the entire source lies within the uniform acceptance region of the photometer. Obviously, photometric point sources need not be visual point sources.

Luminous Directional Energy per Point (LDE)

LDE is defined as the luminous intensity (in the direction of view) from a single intensification of a single point, integrated over time. For example, a point intensified for 1 microsec will be quite visible in a single flash under typical room viewing conditions when it produces a luminous output of 1 candle-microsec, that is, when the point produces the same luminous output as a standard candle would have produced if it were thumb, the temporal distribution of light from a single intensification can be ignored when either or both:

(a)
(1) the luminous output is confined to 20 msec, and (2) the refresh rate exceeds about $20 \mathrm{~Hz}$. In all these cases, oniy the integrated luminous intensity (total amount of light) resulting from a single intensification need by known. 1 This quantity is called the luminous directional energy per point.

Measurement of Luminous Directional Energy per Point

The strategy of this calibration is to compare a reference source of known luminance with a comparably sized dot pattern on the CRO. The basic tenet of absolute luminance calibrations is to avoid big errors, little errors being unavoidable but of little practical importance in vision. By making the reference source the same size and luminance as the CRO dot pattern, the eye can be used as a null meter. At the least, this is helpful in avoiding gross errors of calibration, and it is convenient for a quick check when a system malfunction is suspected.

The reference source is made from: (1) a surface of known luminance ("photometric brightness"), e.g., $1 \mathrm{fL}$; and (2) an aperture of known area, e.g., a square 1 -in. hole.

Together, the luminous surface and the aperture form a standard source of luminous intensity (Fig. 1). Of course, if a standard source of luminous intensity already is available, it may be used instead, but unequal areas make visual comparison unreliable.
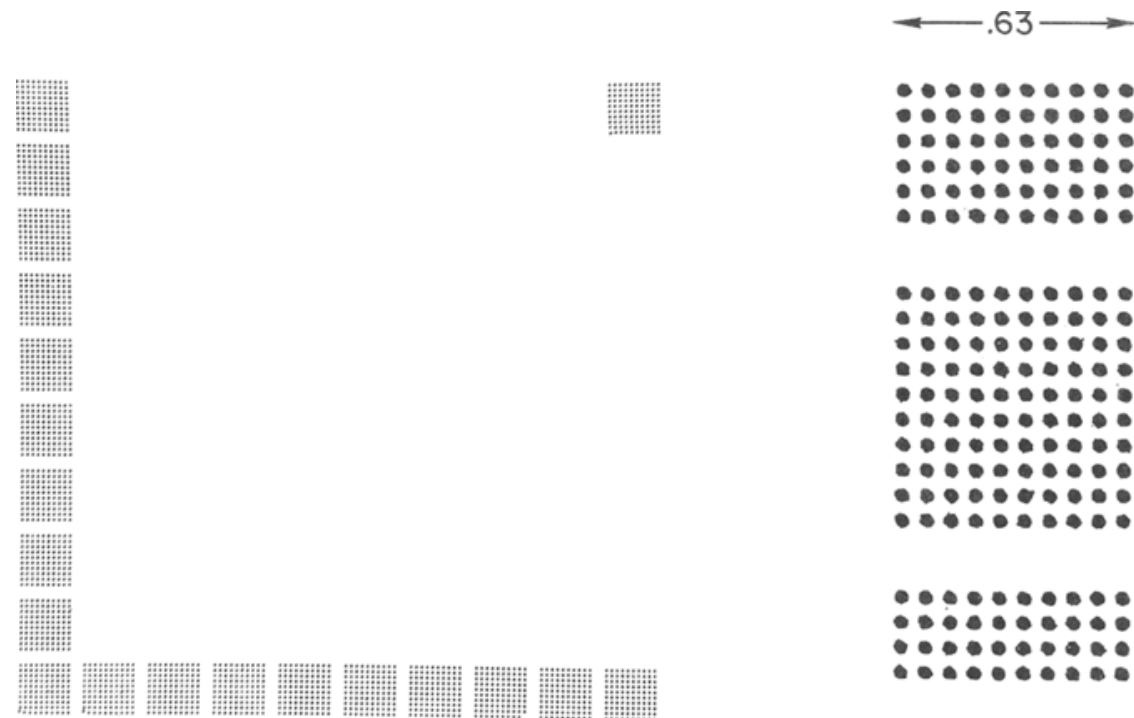

$-\bullet \bullet \bullet$ $\bullet \bullet \bullet \bullet \bullet \bullet \bullet$ $\bullet \bullet \bullet \bullet \bullet \bullet \bullet$ $\bullet \bullet \bullet$

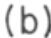

Fig. 2. (a) A negative picture of a CRO test pattern. Each individual square is $5 / 8$ in. wide and composed of a 10 by 10 point matrix. (b) Enlarged detail showing point structure; spot diameter is about 0.03 in. 
Note that a perfectly reflecting-diffusing surface (approximated by white paper), placed $1 \mathrm{ft}$ away from a standard candle (candela) will have a luminance of $1 \mathrm{fL}$. A surface may also be considered as a source of light. The 1-fL surface emits $\pi^{-1} \mathrm{~cd} / \mathrm{ft}^{2}$ (when considered as a point source, i.e., when viewed perpendicularly from afar) so that a 1 -in, square area is equivalent to

$\frac{1 \mathrm{~cd}}{\pi \mathrm{ft}^{2}} \times \frac{1 \mathrm{ft}^{2}}{144 \mathrm{in.}^{2}} \times 1$ in. $^{2}$

$$
=2.210 \times 10^{-3} \mathrm{~cd}
$$

\section{The CRO Calibration Display}

Figure 2 shows a convenient calibration display composed of 20 "squares," each square consisting of a 10 by 10 point pattern. Because of its lateral and vertical extent, the display in Fig. 2 also is convenient for calibrating the $x^{-}$and $y$-axis scale amplifiers. The display is refreshed $n$ times per second. An aperture is used to exclude all except one 10 by 10 square from view.

\section{Photometer}

A null meter can be used to test the equivalence of the luminous intensity from the CRO aperture and the reference surface aperture. The measurement must be made at the same distance from each surface! of course, a photometer with a continuous scale will make the comparison easier. All the light from the reference source must enter the uniform acceptance area of the photometer, and so must all the light from the test aperture (Fig. 1). The refresh rate should be fast enough to give a steady reading on the meter; usually, $20 \mathrm{~Hz}$ will suffice.

From the ratio (or equivalence) of the luminous intensity of $m$ points $X n$ exposures per second to the luminous intensity of the reference aperture, the luminous energy in one exposure of one point is readily calculated. Since luminous units can be very confusing, an example is given here. When the area of the reference aperture is a sq in., and the luminance of the reference surface is $b \mathrm{fL}$, and there are $\mathrm{m}$ points exposed $\mathrm{n}$ times per second, the luminous directional energy per point, $\mathrm{e}$, is

$$
\mathrm{e}=2210(\mathrm{ab}) /(\mathrm{mn}) \text { candle-microsec. }
$$

Application to Vectors, Scan Lines, and Characters

The luminous calibration of vectors and of scan lines is essentially similar to that of points. The critical quantity is the LDE per unit line length. For example, the 100 points of Fig. $2 b$ can be replaced by 10 parallel 1 -in. vectors within the aperture or by 101 -in. segments of scan lines. From the calibration, one obtains the LDE in 10 in. of line, i.e., the LDE per inch.

The LDE per character can be measured by substituting characters for points in the determination of the LDE. For many purposes it is also useful to know the ratio of LDE per character to LDE per point and to LDE per unit line length.

\section{High Refresh Rates}

When viewing of CRO displays is restricted to such high refresh rates that apparent flicker is negligible, then they may be treated as "continuous" displays. In this case, the critical luminous quantities are luminous intensity per point and luminous intensity per unit line length. These intensity quantities are defined identically to the corresponding energy quantities except in that the factor of time is implicit. The point units are candelas or microcandelas, the line units are candelas per inch or microcandelas per inch.

\section{HOW TO USE LUMINOUS DIRECTIONAL ENERGIES} PER POINT AND PER LINE

When a field is filled uniformly and densely with points or scan lines, it appears similar to a continuously illuminated surface, and its intensity is appropriately characterized by its luminance (photometric "brightness"). Luminance is defined as the luminous intensity per unit area. (Equivalently, luminance can be defined by a perfectly reflecting-diffusing surface illuminated by a known luminous flux.) Luminance measurements are particularly useful because they do not depend on the distance from which the surface is viewed, and this distance need not be known. (The intensity calibrations required careful equating of distance.)

The luminance of a CRO display depends both on the LDE per point (or LDE per line) and on the number of points per unit area per unit time (or on the number of inches of line per unit area per unit time). Once the LDEs per point and per line are known, it is merely a matter of multiplication to calculate luminance. For example, a uniform surface of $1 \mathrm{fL}$ luminance emits $\pi^{-1} \mathrm{~cd} / \mathrm{ft}^{2}$. If 1 sq ft of display surface is filled with enough points or lines to appear approximately uniform, if they are refreshed at $\geqslant 20 \mathrm{~Hz}$, and if the total point energy from all the intensifications is $\pi^{-1} \mathrm{~cd}-\mathrm{sec}$, then the display surface will appear to have a luminance of $1 \mathrm{fL}$. When the refresh frequency is doubled, the same array of points will appear to have a luminance of $2 \mathrm{fL}$ because it now produces twice as much light per unit area.

To convert to foot-lamberts from the total candle-microseconds produced by all point or line intensifications within an area, $a$, in $1 \mathrm{sec}$, one needs merely to reverse Eq. 2, i.e.,

$$
b=0.00452 \mathrm{e} / \mathrm{a}
$$

where b is luminance in foot-lamberts, $e$ is the LDE in candle-microseconds, and $a$ is the area in square inches.

\section{LINEARITY PROBLEMS}

An important characteristic of phosphors to consider in luminance calibrations is that their response can be nonlinear and can depend on how frequently, how intensely, and how many neighboring points are intensified. However, a phosphor becomes more linear the less output is required within any small area in any small time, so it is possible to set up conditions under which the response is approximately linear. The purpose of the 20 squares in the calibration display of Fig. 1 is to provide ready comparison with calibration displays having only 10 or 2 squares in all, and repeated at proportionately greater rates, i.e., producing correspondingly greater output in the remaining square seen through the aperture. When the response of the phosphor is being measured in the linear range, then halving the refresh rate should exactly halve the luminous flux output through the aperture. This halving should be tested when calibrating luminous energies.

Linearity considerations are important when it is necessary to calibrate a display whose intensity is set to a level high enough to permit clear visibility of a single flash. In this case, refreshing the display as frequently as 20 times per second might damage the phosphor. This danger can be ameliorated by intensifying different points-within the same aperture-on successive exposures. The problem does not arise in calibrating continuously refreshed displays. In this case, the calibration refresh rate should be chosen the same as the viewing refresh rate.

One word of advice: Never trust calculations more than necessary. For example, though luminance is readily calculated from LDEs, it also is easily measured directly. Measure it to confirm the calculation. Finally, computers usually generate frames so rapidly that the number and timing of frames cannot be checked by eye. Before considering a display program finished, it always is worthwhile to use 
a photocell and an oscilloscope to monitor the display and to verify that the program is generating the intended number of frames in the intended sequence.

\section{REFERENCES}

LEGRAND. Y. Light, colour and vision. (2nd ed., translated from French.) London: Chapman \& Hall, 1968.

WALSH, J. W. T. Photometry. (2nd ed.) London: Constable, 1953.
NOTE

1. There is no conventionally agreed upon photometric term for the integral of luminous intensity (LeGrand, 1968; Walsh, 1953 ); it is the quantity of light per solid angle, emitted in a particular direction. Possible units are (lumens $x$ seconds)/steradian, talbots/steradian, and candles $x$ seconds. Integrated luminous intensity is perhaps the most accurate name, but luminous directional energy better carries the meaning in the present context. In any case, the choice of units (candles $x$ microseconds) makes it perfectly clear what is being measured.

\title{
Flicker in computer-generated visual displays: Selecting a CRO phosphor and other problems
}

\author{
GEORGE SPERLING $\dagger$ \\ Bell Telephone Laboratories, Murray Hill, New Jersey 07974 \\ and \\ New York University, New York, N.Y. 10003
}

The visual system's response to flicker is approximated by 6-9 RC stages in series. The CRO phosphor can be represented by one additional RC stage. Therefore, increasing the refresh rate by a factor of $\mathrm{k}$ can be $\mathrm{k}^{5}$ to $\mathrm{k}^{8}$ times more effective in reducing apparent flicker than increasing the phosphor time constant. "Slow" phosphors impair the display of rapid movement and cause undesirable persistence of old picture contents after they have been altered. Behavioral scientists usually should choose fast, efficient phosphors. Display programs should be written so that spatially adjacent points of a display are intensified as close together in time as possible.

A visual display on a cathode ray oscilloscope is composed of points, vectors, characters, or scan lines. A single picture (frame) can be painted in a relatively short time, depending, of course, on the number of elements of which it is composed, as elements are painted sequentially. In order to give the viewer the illusion that the display is present continually, the frame must be repainted (refreshed) about 20 to 40 times per second. The more frequently it is refreshed, the less it appears to flicker.

In contemporary CRO displays, each individual point (of a frame) typically is electronically pulsed (intensified) for 1 microsec or less. At a refresh rate of 40 frames/sec, the point will be reintensified 40 times per second, 1.e., every 25 msec. The transduction of the electron intensification beam to light is accomplished by a phosphor painted on the inside of the CRO tube.

*Presented at the North Atlantic Treaty Organization, Advanced Studies Institute on: On-Line Computing for Behavioral Science, Department of Psychology. Sheffield, England, July 19, 1969.

tRequests for reprints should be sent to: G. Sperling, Room 2D518, Bell Telephone Laboratories, Murray Hill, New Jersey 07974 .
Depending on the particular phosphor, the light output in response to the brief intensification pulse may either persist long after the pulse or it may follow the shape of the intensification pulse. The phosphor is a means of prolonging, or "spreading out," the light in time and thereby reducing the apparent flicker.

The light output of a phosphor in response to an exceedingly brief intensification pulse (impulse) typically follows an exponential curve of the form illustrated in Fig. 1. This exponential decay curve is characterized by a single parameter, its decay time constant $(\tau)$, which is defined as the time taken to decay to .368 of the initial value. ${ }^{1}$ When the time constant is long, apparent flicker is minimized; when it is short, flicker is maximized.

\section{THE PHOSPHOR PROBLEM:} FAST OR SLOW?

In "continuous" CRO displays it is desirable to minimize apparent flicker. This could be accomplished either by (1) choosing a phosphor with a long time constant (a "slow" phosphor), or by $(2)$ increasing the refresh rate.

One disadvantage of choosing a phosphor with a long time constant is that it makes the display system unsuitable for applications requiring brief visual presentations, because brief exposures are impossible with slow phosphors. Furthermore, when the display contents are altered, a slow phosphor causes the old contents to persist visibly under the new picture. This difficulty makes slow phosphors unsuitable for presenting rapid motion.

Alternatively, apparent flicker can be reduced by increasing the refresh rate. However, it may be impossible to increase the refresh rate when frames are composed of large numbers of points or characters. Even when it is possible, a high refresh rate places an added burden on the computer. The following sections evaluate these factors in terms of the flicker sensitivity of the human visual system. The conclusion (for behavioral scientists) will be that phosphors with short time constants should be chosen, except when display systems are dedicated to static displays.

Flicker Sensitivity of the Visual System

The response of the visual system to flickering light can be predicted from the model illustrated in Fig. 2. It is a good first-order mathematical approximation to a more complicated model (Sperling \& Sondhi, 1968) in the frequency range of interest, i.e. for frequencies $\geqslant 10 \mathrm{~Hz}$. The basic elements of this approximation are RC stages, linear elements whose response to an impulse input is an exponential output of the form already described in Fig. 1.2

In the dark, the response of the visual system to flicker can be represented by three $\mathrm{RC}$ stages in series (each having a time constant of about $25 \mathrm{msec}$ ) plus six more RC stages in series (each having a time constant of about $5 \mathrm{msec}$ ). In very bright light, the visual system's response to flicker is represented simply by the final six stages (each with $\tau=5 \mathrm{msec}$ ). That is, in very

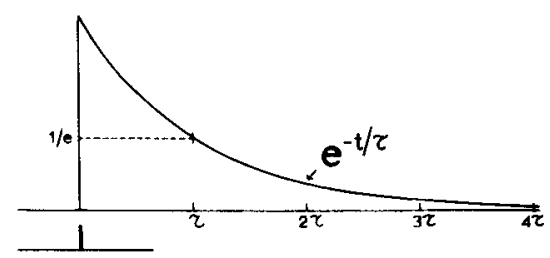

Fig. 1. Light response of a phosphor to an intensification impulse (theoretical). Ordinate: light output; abscissa: time; the intensification impulse is indicated below. The response illustrated is an exponential decay function with a time constant of $\tau$. 